\title{
Spatial position and posture of component detection system based on binocular stereo vision
}

\author{
Liuyuan $^{1, a^{*}}$ Feiyunfeng ${ }^{2, b}$ Wang wei $^{1, c}$ Songyinhao ${ }^{2, d}$ \\ ${ }^{1}$ Xi'an Research Institute of High Technology, Xi'an 710025, Shaanxi Province, China \\ 2 Beijing Special Engineering Design Institute, Beijing 100011, China \\ aliuyuanbixue@163.com, bchengxi913@163.com, cxiangxuanstudy@sina.cn, d418394491@qq.com
}

Keywords: binocular stereo vision, position and posture detection, automatic docking

Abstract. With the continuous improvement of the manufacturing level in the aerospace, automobile, shipbuilding and other fields, higher requirements for the assembly precision and speed of the components in the manufacturing process are raised. However, the manual operation method is generally adopted in the work of large mechanical parts assembly in our country. Through the visual feedback of the human eye to the operation interface, the labor intensity is large, the efficiency is low, and the assembly cycle is long. To solve this problem, spatial position and posture of object detection system based on binocular stereo vision is proposed. On the basis of the predecessors, the traditional calibration method is improved, the calibration of the binocular camera is realized and the position and position deviation of the component is measured by the binocular camera. The difference of the position detection system of the binocular vision is less than $0.1 \mathrm{~mm}$ in the three space positions, and the difference between the three rotation angles is less than 0.15 degrees. It lays the foundation for automatic docking of components.

\section{Introduction}

In recent years, the level of aerospace, automotive, shipbuilding and other fields, which is closely related to the manufacturing industry, has developed rapidly in the design and manufacture of large mechanical parts. With the continuous improvement of the factory manufacturing level, the assembly accuracy and speed of components in the manufacturing process are higher. But at present, the work of large mechanical parts assembly in our country mainly depends on manual completion, that is to put each part on the bracket or trailer platform first, then according to the bolts and screw holes at different positions on the combination surface of each component, and rely on a number of workers to push one of the parts slowly close to another part, and pass through continuously. Observe and adjust the relative position of bolt and screw hole to complete the alignment and insertion operation of components. Moreover, the docking effect of this way often depends on the experience and operation level of the personnel. The docking accuracy is low, the installation speed is slow, and the operation stability is poor.

With the development of machine vision measurement technology ${ }^{[1-3]}$, laser tracking measurement technology ${ }^{[4,5]}$ and robot technology ${ }^{[6]}$ and so on, automatic docking has become an inevitable trend of development ${ }^{[7]}$, in the docking of large and irregular large mechanical parts. Binocular vision is an important form of machine vision. It is simple, reliable, flexible and widely used. In this paper, binocular vision position and position detection technology is used to propose a binocular vision component space pose detection method, which lays the foundation for automatic docking of components.

\section{Construction of binocular vision position detection system}

\section{Structure model of binocular stereo vision system}

The structure model of the binocular vision system is shown in Fig. $1 .{ }^{0_{1}}$ and $^{0_{2}}$ are the optical centers of lenses 1 and 2 respectively. The distance between them is called the baseline and 
represented by B. ${ }^{0_{1}}$ and ${ }^{0_{2}}$ are the center of the left and right image planes, respectively. Set two CCD camera horizontally, use CCD1 and CCD2 to represent the image plane of the left and right cameras respectively, and the left and right image coordinate systems are $O_{1}-X_{1} Y_{1}$ and $O_{2}-X_{2} Y_{2}$ respectively. $\mathrm{O}_{1} \mathrm{O}_{1}$ is the left camera axis and $\mathrm{O}_{2} \mathrm{O}_{2}$ is the right camera axis. The spatial points $\mathrm{P}$ are respectively imaging on the left and right image plane, and the coordinates corresponding to the image points are $P_{1}\left(X_{1}, Y_{1}\right)$ and $P_{2}\left(X_{2}, Y_{2}\right)$ respectively. It is now used $o_{1}-x y z$ as the coordinate system of the binocular system. The effective focal length of the camera is $f_{1}$ and $f_{2}$ respectively. The angle of the optical axis and the baseline are $\alpha_{1}$ and $\alpha_{2}$ respectively. The horizontal projection angles of the space point $\mathrm{P}$ are $\omega_{1}$ and $\omega_{2}$ respectively. The vertical projection angles are $\phi_{1}$ and $\phi_{2}$ respectively. According to the geometric structure relations, the coordinates of the $\mathrm{P}$ points can be obtained as follows:

$$
\begin{aligned}
& \left\{\begin{array}{c}
x=\frac{B \cot \theta_{1}}{\cot \theta_{1}+\cot \theta_{2}} \\
y=\frac{z \tan \phi_{1}}{\sin \theta_{1}}=\frac{z \tan \phi_{2}}{\sin \theta_{2}} \\
z=\frac{B}{\cot \theta_{1}+\cot \theta_{2}}
\end{array}\right. \\
& \theta_{1}=\alpha_{1}+\omega_{1}, \quad \theta_{2}=\alpha_{2}+\omega_{2} \\
& \tan \phi_{1}=Y_{1} / \cos \omega_{1}, \quad \tan \phi_{2}=Y_{2} / \cos \omega_{2} \\
& \omega_{1}=\arctan \left(X_{1} / f_{1}\right), \quad \omega_{2}=\arctan \left(X_{2} / f_{2}\right)
\end{aligned}
$$

The structure parameters of the system constitute the important parameters of the system. By analyzing the error composition of the above formula and the precision requirements of the actual assembly, the configuration of the binocular system can be determined.

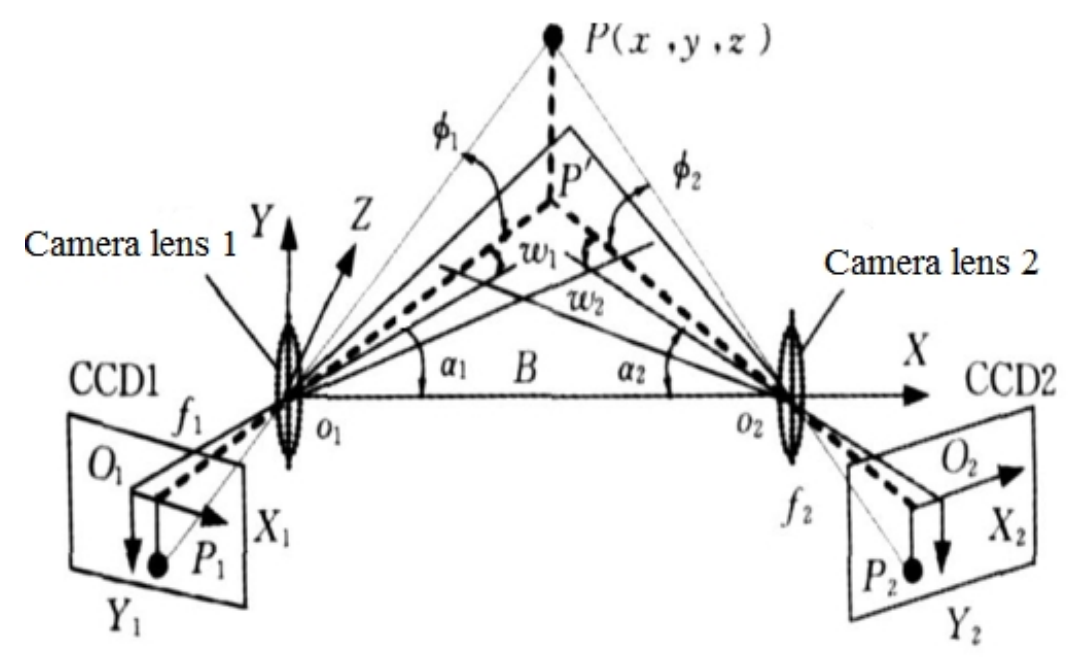

Fig. 1 Structure Model of binocular stereo vision system

\section{Experimental verification}

Based on the above theoretical analysis, we build a dual vision component space pose detection system. The main equipment is: industrial camera (Microvision MV-EM200M), industrial lens (Computar M1614- MP2), MITSUBISHI manipulator (RV-3SQ), linear guide rail (single degree of freedom guide, precision $=0.01 \mathrm{~mm})$. According to the equations $(1)$ to (4), the camera has 
symmetrical distribution, the camera's baseline distance is $360 \mathrm{~mm}$, and the angle between the camera and the baseline is $67.5^{\circ}$.

\section{The realization of calibration}

The traditional camera calibration method can be used for any camera model with high calibration accuracy, but the calibration process is complex and requires expensive high precision calibration equipment. Zhang has proposed a simple, flexible and practical camera calibration method ${ }^{[8]}$. This paper has made improvements on the basis of previous predecessors, and realized the calibration of the binocular camera. The total size of the calibration board is $100 \mathrm{~mm}^{*} 100 \mathrm{~mm}$, the checkerboard formation area is $70 \mathrm{~mm} * 60 \mathrm{~mm}$, and the checkerboard size is $10 \mathrm{~mm} * 10 \mathrm{~mm}$, the error is $20 \mu \mathrm{m}$. There are 30 inner corners in the whole checkerboard. Before each shot, it is necessary to change the position and posture of the calibration board until 20 pictures are taken, then the calibration button on the interface is used to get the inside and outside parameters of the camera, and the calibration work is completed. The camera calibration interface is shown in Fig. 2.

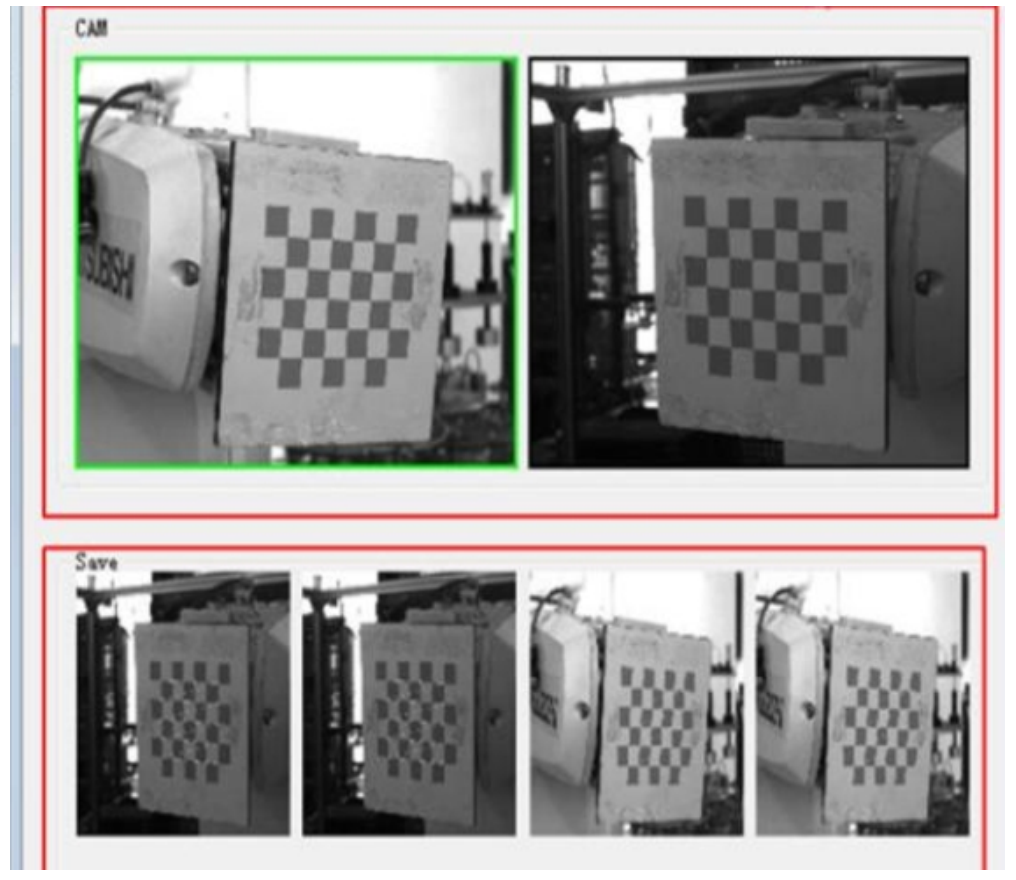

Fig. 2 The camera calibration interface

After calibration, the internal matrices of camera 1 and camera 2 are (5) and (6) respectively, and the binocular matrix $\mathrm{R}$ and $\mathrm{T}$ matrix are (7) and (8) respectively.

$$
\begin{aligned}
R_{1} & =\left[\begin{array}{l}
3717.66,0,788.13 \\
0,3717.35,627.715 \\
0,0,1
\end{array}\right] \\
R_{2} & =\left[\begin{array}{l}
3735.48,0,767.973 \\
0,3734.93,604.172 \\
0,0,1
\end{array}\right] \\
R & =\left[\begin{array}{l}
-0.03079904083105934,0.007236546355386415,0.9994994004404071 \\
0.01628958022339765,0.9998446185183378,-0.006737091068492179 \\
-0.9993928500144637,0.01607392972381081,-0.03091213553285763
\end{array}\right]
\end{aligned}
$$




$$
T=\left[\begin{array}{l}
-216.8018448703145 \\
1.314678751945799 \\
233.6980553202565
\end{array}\right]
$$

\section{Measurement component of position and posture error of six degrees of freedom in space}

In order to verify the measurement accuracy of the position and posture detection system based on binocular vision, the error of the measurement results on the space six degree of freedom is calculated by the experimental method. The error distribution of binocular measuring system in three directions of $\mathrm{x}, \mathrm{y}$ and $\mathrm{z}$ is drawn by comparison of linear guide rail (single degree of freedom guide rail and precision $=0.01 \mathrm{~mm}$ ). The error of the binocular measurement system on three rotational degrees of freedom (pitch angle, roll angle, yaw angle) is used in the experiment. In the experiment, the posture of the calibrated plate is used as the detection object, and the result of the posture calculation of the calibrated plate obtained by the high precision manipulator is taken as the reference value, and the binocular vision is compared with the binocular vision measurement results. After 100 time measurements, the difference of the position detection system of the binocular vision is less than $0.1 \mathrm{~mm}$ in the three space positions, and the difference between the three rotation angles is less than 0.15 degrees. Fig. 3 is the experimental scene when the error is measured. Fig. 4 is the result of the test.

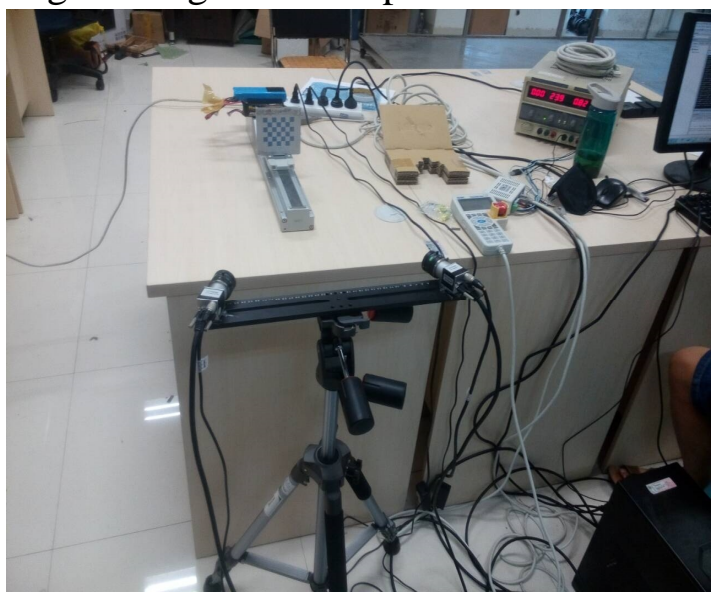

(a)Position error detection

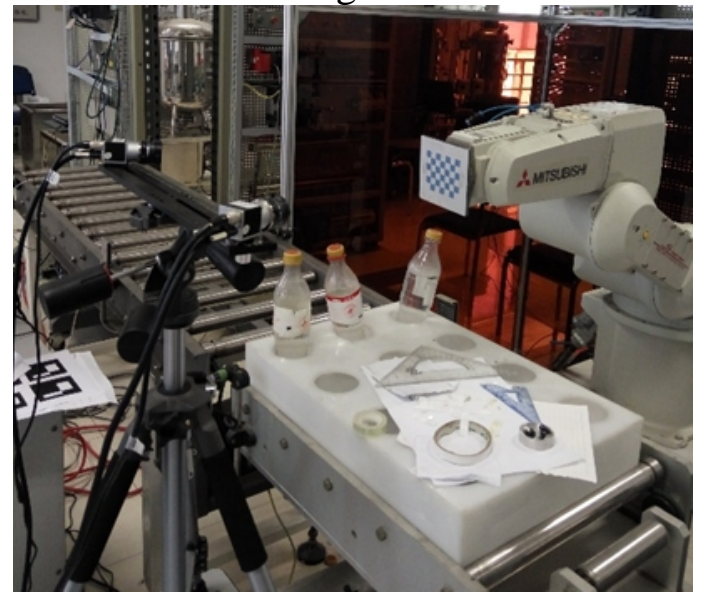

(b) Posture error detection

Fig. 3 The error measured experimental scene
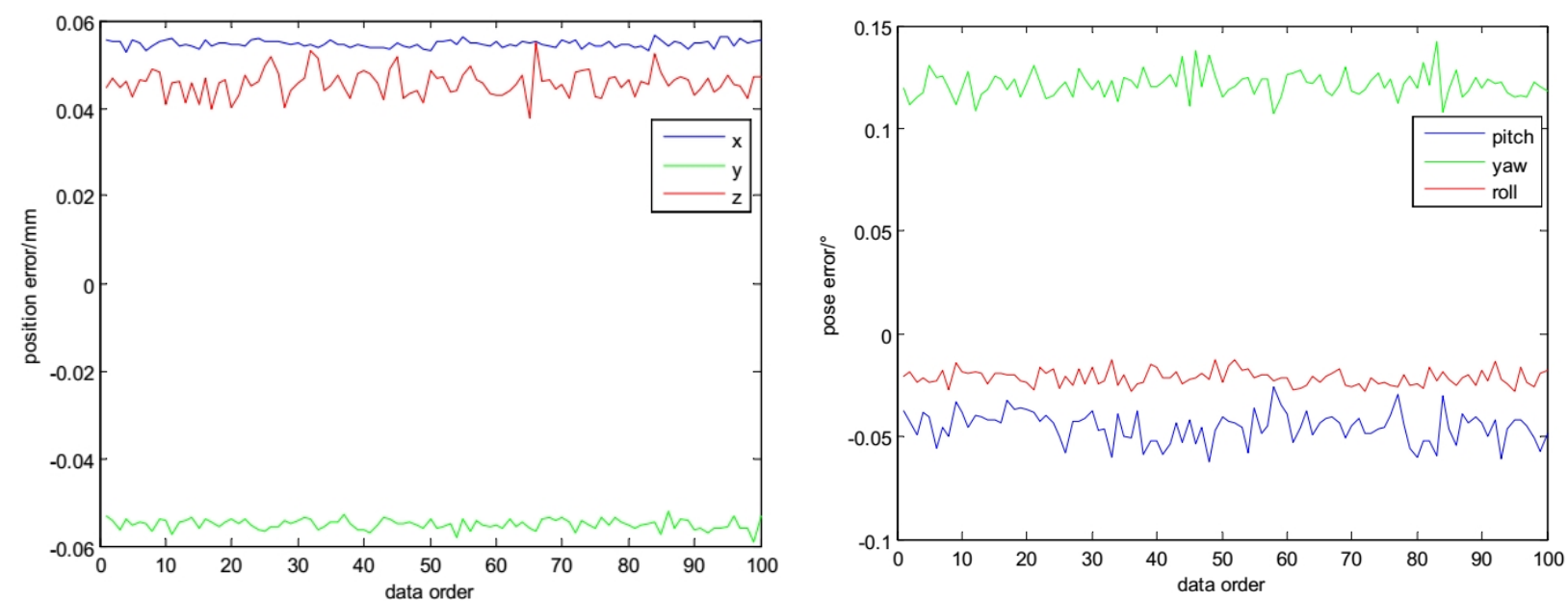

Fig. 4 The error detection result of the position and posture.

\section{Conclusion}

This paper makes a theoretical study and experiment on the position and posture detection system based on binocular stereo vision. The difference of the position detection system of the binocular vision is less than $0.1 \mathrm{~mm}$ in the three space positions, and the difference between the three rotation 
angles is less than 0.15 degrees. It is proved that the design method of the system is simple and the structure is easy to be realized. It can detect the position and pose of the components in high precision, which has a good prospect of application.

\section{Reference}

[1] Chen X. N., Guo J., Yao Y. Y. Sichuan Journal of ordnance industry, 34 (2013),P.99 (in Chinese).

[2] Gou W. T. Xie W. Q. Journal of Ordnance Equipment Engineering, 2 (2016),P.105 (in Chinese).

[3] Li J. Journal of Modern Optics, 39 (2017),P.1.

[4] Li J. Q., Zhao R. H., Chen J. L., et al. Iet Science Measurement \& Technology, 10(2016),P.704.

[5] Cattini S., Rovati L. Instrumentation and Measurement Technology Conference Proceedings. IEEE, 2016, P.1.

[6] Mergler H. IEEE Journal on Robotics \& Automation, 3(2003),P.87.

[7] Huang G. Q., Mak K.L. Computers in Industry ,38(1999),P.17.

[8]Lin D.M. Zhang A., Wang P., et al. Journal of Lanzhou University of Technology, 42 (2016),P.78 (in Chinese) 